\title{
On the Tomographic Reconstruction of the 3D Electron Density for the Solar Corona from STEREO COR1 Data
}

\author{
M. Kramar • S. Jones · J. Davila • B. Inhester • \\ M. Mierla
}

Received: 8 December 2008 / Accepted: 16 June 2009 / Published online: 4 July 2009

(C) The Author(s) 2009. This article is published with open access at Springerlink.com

\begin{abstract}
We present for the first time a three-dimensional reconstruction of the electron density in the corona at distances from $1.5 R_{\odot}$ to $4 R_{\odot}$ using COR 1 STEREO observations. The reconstruction is performed using a regularized tomography inversion method for two biweekly periods corresponding to Carrington Rotations 2058 and 2066. Images from the two STEREO spacecraft are used to compare the reconstructed density structures with coronal features located by triangulation. We find that the location of a bright tip of a helmet streamer obtained from the tomographic reconstruction is in good agreement with the location obtained by triangulation. The reconstructed density structure of the equatorial streamer belt is largely consistent with the variation of the current sheet derived from a potential magnetic field extrapolation for most of the equatorial region and for an MHD model of the corona. A zero-value density region in the reconstruction is identified with a low-density region seen in an EUVI image below the reconstruction domain.
\end{abstract}

STEREO Science Results at Solar Minimum

Guest Editors: Eric R. Christian, Michael L. Kaiser, Therese A. Kucera, O.C. St Cyr.

M. Kramar $(\bowtie) \cdot$ M. Mierla

Physics Dept., The Catholic University of America, 620 Michigan Ave NE, Washington, DC 20064, USA

e-mail: kramar@helio.gsfc.nasa.gov

S. Jones

Physics Dept., University of Maryland, 082 Regents Dr., College Park, MD 20742, USA

S. Jones · J. Davila

NASA Goddard Space Flight Center, Code 671, Greenbelt, MD 20771, USA

B. Inhester

Max-Planck-Institut für Sonnensystemforschung, 37191 Katlenburg-Lindau, Germany

M. Mierla

Royal Observatory of Belgium, Ringlaan 3, 1180 Brussels, Belgium

M. Mierla

Astronomical Institute of the Romanian Academy, 040557 Bucharest, Romania 
Keywords Sun: corona $\cdot$ Sun: magnetic fields $\cdot$ Methods: data analysis $\cdot$ Techniques: tomography $\cdot$ Techniques: image processing

\section{Introduction}

To understand physical processes in the solar corona, it is necessary to measure the properties of the coronal plasma. One of the most fundamental properties is the distribution of the electron density. However, the solar corona is optically thin, so in coronagraph images the radiation coming from the corona is integrated over the observer's line of sight (LOS), and it is impossible to localize any structure in the corona with an observation from only one viewing direction. The simultaneous Solar Terrestrial Relations Observatory (STEREO) observations (from two viewing directions) allow localization in three-dimensional space of pointlike or linelike structures by triangulation, but the structure of extended objects cannot be derived by this method (Inhester, 2006).

To reconstruct extended structures in the optically thin corona, it is necessary to have observations from more than two directions. This is the essence of tomography. In practice, a rigid rotation of the coronal density structures is usually assumed. Coronagraph data from half a solar rotation then are necessary as input to the reconstruction algorithm, and only structures that are stationary over about 14 days can reliably be reconstructed (Davila, 1994; Zidowitz, 1999; Frazin and Kamalabadi, 2005).

The tomographic technique for reconstructing the solar corona was previously studied by Davila (1994). Panasyuk et al. (1998) applied the tomographic technique to UVCS/SOHO data. Zidowitz (1999) made a tomographic reconstruction of the corona at distances from $1.3 R_{\odot}$ to $1.9 R_{\odot}$ using the Mauna Loa Solar Observatory (MLSO) Mark-III K-coronameter. Frazin and Janzen (2002) and Frazin et al. (2007) used SOHO LASCO/C2 data for a reconstruction that covers the coronal region from $2.4 R_{\odot}$ to $6 R_{\odot}$.

The COR1 coronagraphs onboard the STEREO spacecraft routinely provide images of the corona at heights from $\sim 1.5 R_{\odot}$ to $4 R_{\odot}$ where the transition from closed to open coronal structures occurs for the first time. Previously, this coronal region was partially covered only by observations from Earth such as by the MLSO MK-IV coronagraph.

Because STEREO has two spacecraft observing from different viewing directions, we can reduce the observational period (and as a consequence the duration of the stationary structures to be reconstructed) down to about a week when the spacecraft reach their angular separation of $90^{\circ}$. This separation had not yet been reached at the time this paper was prepared. For this reason we use data only from one spacecraft, namely, STEREO-B. However, by using triangulation we can check the correctness of the tomographic reconstruction at least for some coronal structures that are not spatially extended.

The paper is organized as follows. In Section 2, the LOS integration kernel and the inversion procedure is described. Section 3 describes the procedure for subtracting the scattered light from the image to obtain the coronal intensity. Section 4 presents the reconstructed electron density for the period corresponding to Carrington Rotation (CR) 2058. For this reconstruction, an independent validation of some reconstructed structures was made by using the simpler triangulation. Also, the reconstructed density is compared with existing coronal models. 


\section{Method}

\subsection{Thompson Scattering}

In the low corona (below $\sim 10 R_{\odot}$ ), the polarized brightness $(\mathrm{pB})$ is dominated by the electron K corona (Blackwell and Petford, 1966a, 1966a; Moran et al., 2006; Frazin et al., $2007)$. So, the intensity of the polarized brightness $\left(I_{\mathrm{pB}}\right)$ signal as a fraction of the mean solar brightness is

$$
I_{\mathrm{pB}}\left(\hat{\mathbf{e}}_{\mathrm{LOS}}, \boldsymbol{\rho}\right)=\int_{\text {LOS }} K(\mathbf{r}) \cdot N_{\mathrm{e}}(\mathbf{r}) \mathrm{d} \ell,
$$

where $N_{\mathrm{e}}$ is electron density and $\rho$ is a vector in the plane of the sky (POS) from the Sun's center to the lines of sight and perpendicular to the LOS. The kernel function $K$ is defined by the Thompson scattering effect (Van de Hulst, 1950; Billings, 1966; Quemerais and Lamy, 2002):

$$
K=\frac{\pi \sigma}{2\left(1-\frac{u}{3}\right)}[(1-u) A(r)+u B(r)] \frac{\rho^{2}}{r^{2}},
$$

where expressions for $A(r)$ and $B(r)$ are the same as in Quemerais and Lamy (2002), $\sigma=7.95 \times 10^{-26} \mathrm{~cm}^{2}$ is the Thompson scattering cross section for a single electron, $R_{\odot}$ is the solar radius, and the linear limb-darkening coefficient, $u$, is set to 0.33 in the present calculations.

\subsection{Tomography}

Discretizing the LOS integral (1), we obtain a set of algebraic equations that can be represented by the matrix equation

$$
\boldsymbol{A} \cdot \mathbf{X}=\mathbf{Y}
$$

Here, the elements $x_{j}$ of the column vector $\mathbf{X}$ contain the values of electron density $N_{\mathrm{e}}$ in the grid cells with index $j=1, \ldots, n$, and $y_{i}$ is the data value for the $i$ th ray, where index $i=1, \ldots, m$ accounts for both the view direction $\hat{\mathbf{e}}_{\mathrm{LOS}}$ and pixel position in the image. The matrix element $a_{i j}$ represents the intersection of volume element $j$ with the LOS related to pixel $i$, multiplied by the kernel function $K(\mathbf{r})$.

To minimize the effects of noise and data gaps, we use regularization (Tikhonov, 1963) and minimize the function

$$
F=|\boldsymbol{A} \cdot \mathbf{X}-\mathbf{Y}|^{2}+\mu|\boldsymbol{R} \cdot \mathbf{X}|^{2},
$$

where matrix $\boldsymbol{R}$ is a diagonal-like matrix such that the second term in Equation (4) is the first-order smoothing term, that is, the square difference in value between two neighboring grid cells, summed over all cells. The regularization parameter, $\mu$, regulates balance between the smoothness of the solution on one hand and the noise and reconstruction artifacts on the other. The result of the inversion depends on a number of factors, including the number of iterations and value of $\mu$.

For the reconstructions to follow, the value of $\mu$ was chosen by using the cross-validation method (Frazin and Janzen, 2002). The iterations are performed until the first term in Equation (3) becomes slightly less than the data noise level, which is essentially the Poisson noise in the data. 


\subsection{Inversion}

For ray tracing during the calculation of the matrix $\boldsymbol{A}$, we use an approximation in which we assume all rays to be parallel for the same image. At $2 R_{\odot}$ the error is comparable to or less than the cell size in the reconstruction, which used a $128^{3}$ rectangular grid within a $4 R_{\odot}$ sphere.

The coronal electron density drops very rapidly with distance from the Sun, introducing a large dynamic range in the data, which causes linear artifacts in the reconstruction. So to increase the contribution of signals from those LOSs that pass through the low-density regions, and to reduce the artifacts in the numerical reconstruction at larger distances from the Sun, a weighting function

$$
w_{i}=\frac{1}{\left(y_{i}^{(\mathrm{FT} 1)}\right)^{2}}
$$

was used for the first term in Equation (4). Here, $y_{i}^{(\mathrm{FT} 1)}$ is the inverse Fourier transform of the function $y_{i}\left(r_{\mathrm{p}}, \phi_{\mathrm{p}}\right)$ on $\phi_{\mathrm{p}}$ with harmonics taken up to first order, where $y_{i}\left(r_{\mathrm{p}}, \phi_{\mathrm{p}}\right)$ is the data value at the position $\left(r_{\mathrm{p}}, \phi_{\mathrm{p}}\right)$ in the polar coordinate system for some particular image. The value of $r_{\mathrm{p}}$ is fixed for a given pixel and equal to the radial distance from the center of the Sun's disk to the pixel. The influence of the weighting factor (5) on the inversion is demonstrated in the Appendix.

The standard conjugate gradient method was used for the inversion with a constant density as starting point for iterations. To ensure positive density values, the negative density values sometimes obtained in the inversion are set to the value of $1 \mathrm{~m}^{-3}$ after every iteration.

\section{Data Preparation}

We use the pB-intensity data from the COR1 instrument onboard the STEREO spacecraft. Because it views the corona close to the limb, the COR1 instrument records a significant amount of scattered light that must be subtracted from the image prior to reconstruction. Details of this subtraction are discussed in the following. After subtracting the scattered light, a median filter was applied to reduce anomalously bright pixels caused by cosmic rays. Then, every third image pixel was taken to reduce the size of matrix $\boldsymbol{A}$ in Equation (3), so the size of the input data images is $341 \times 341$. The calibration of the instrument for solar brightness units is included in Solar Soft IDL routines (Thompson and Reginald, 2008).

Because it is practically impossible to find a two-week period when the corona is absolutely stable and does not show any activity, we exclude all images with CME activity and took only the "stable" images. The resulting image cadence is not precisely constant but averages $1-3$ images per day.

\subsection{Removing the Scattered Light: Monthly versus Roll Minimum}

Proper removal of instrumental scattered light is essential for coronal reconstruction. One of the ways to do this is to subtract a monthly minimum (MM) background. The monthly minimum approximates the instrumental scatter by finding the minimum value of each pixel in all images over roughly a one-month period. However, this method tends to overestimate the scattered light in the streamer belt (equatorial region). For these pixels, their minimal value over a month will contain both the scattered light and the steady intensity value from 

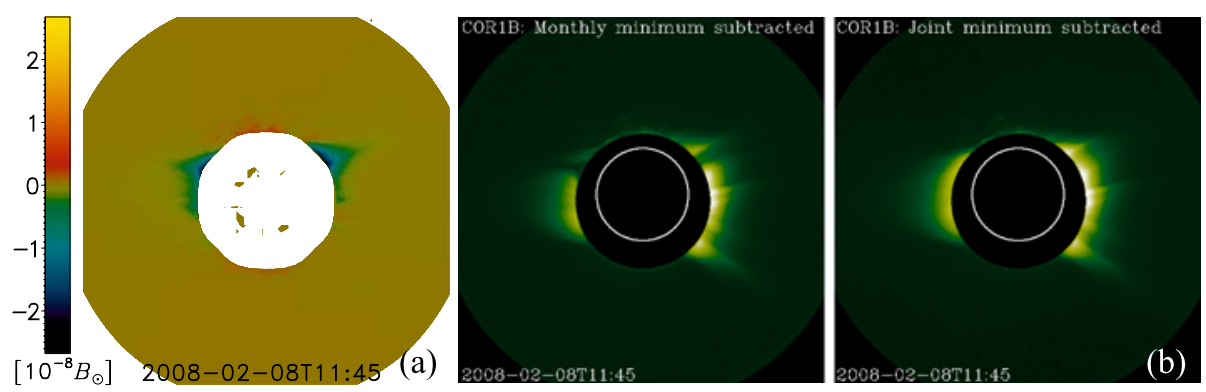

Figure $1 \mathrm{pB}$ image with MM background subtracted minus corresponding image with RM background subtracted (left). pB images with MM background subtracted (middle) and with JM background subtracted (right). All images are from COR1-B and are for 8 February 2008 at 11:45 UT.

the corona. Hence, using such pixels as input for electron density reconstruction, we would obtain an electron density that is lower than the actual density.

Another way to remove the scattered light is to subtract a roll minimum (RM) background. The roll minimum background is the minimum value of each pixel obtained during a roll maneuver of the spacecraft (instrument) around its optical axis. Because the coronal polar regions are much darker than equatorial ones, the minimum value of pixels in the equatorial region during the roll maneuver are nearer to the value of the scattered light intensity than the MM. However, for the polar region, the RM value may not represent the scattered light level, because the roll is usually obtained from images taken at $\sim 45^{\circ}$ finite steps, and some of the polar plumes overlap with others. Therefore, for polar regions, it is better to subtract the MM background because during the rotation of the corona during a month there is a larger probability that most of the pixels in the polar regions will have values corresponding to the polar coronal hole background (i.e., the interplume region).

This is clearly seen in Figure 1(a). This figure shows the difference between a pB image with MM background subtracted and a $\mathrm{pB}$ image with $\mathrm{RM}$ background subtracted. The difference in the polar regions is positive, meaning that in the polar regions pixels values of MM background are lower than those of RM background. Because of this the MM background is closer to the scattered light intensity in the polar regions. The opposite situation is observed in the equatorial region (i.e., pixels values of MM background are larger than those of RM background).

So, the best estimate of scattered light is obtained with a combination of the minimum of roll and MM backgrounds (i.e., a joint minimum (JM)). Figure 1(b) shows two images: one with MM background subtracted and the other with JM background subtracted. Subtle differences exist between these two images. The image with the JM background subtracted is brighter and smoother. Also, one can see a noticeable difference between the images in the upper left sectors where, in the image with MM background subtracted, a dark gap is seen. This indicates that by subtracting the MM in this region, we also subtract a portion of the coronal light.

\subsection{Removing the Scattered Light: Time-Dependent Sensitivity}

The sensitivity of the instrument changes with time, decreasing by about $0.25 \%$ per month (Thompson and Reginald, 2008), and the roll maneuvers are done rather rarely, with four rolls for STEREO-B in 2007 (on 29 and 31 January, 17 April, and 22 October) and two for STEREO-A (on 20 February and 22 October). Therefore it is impossible to use a RM 

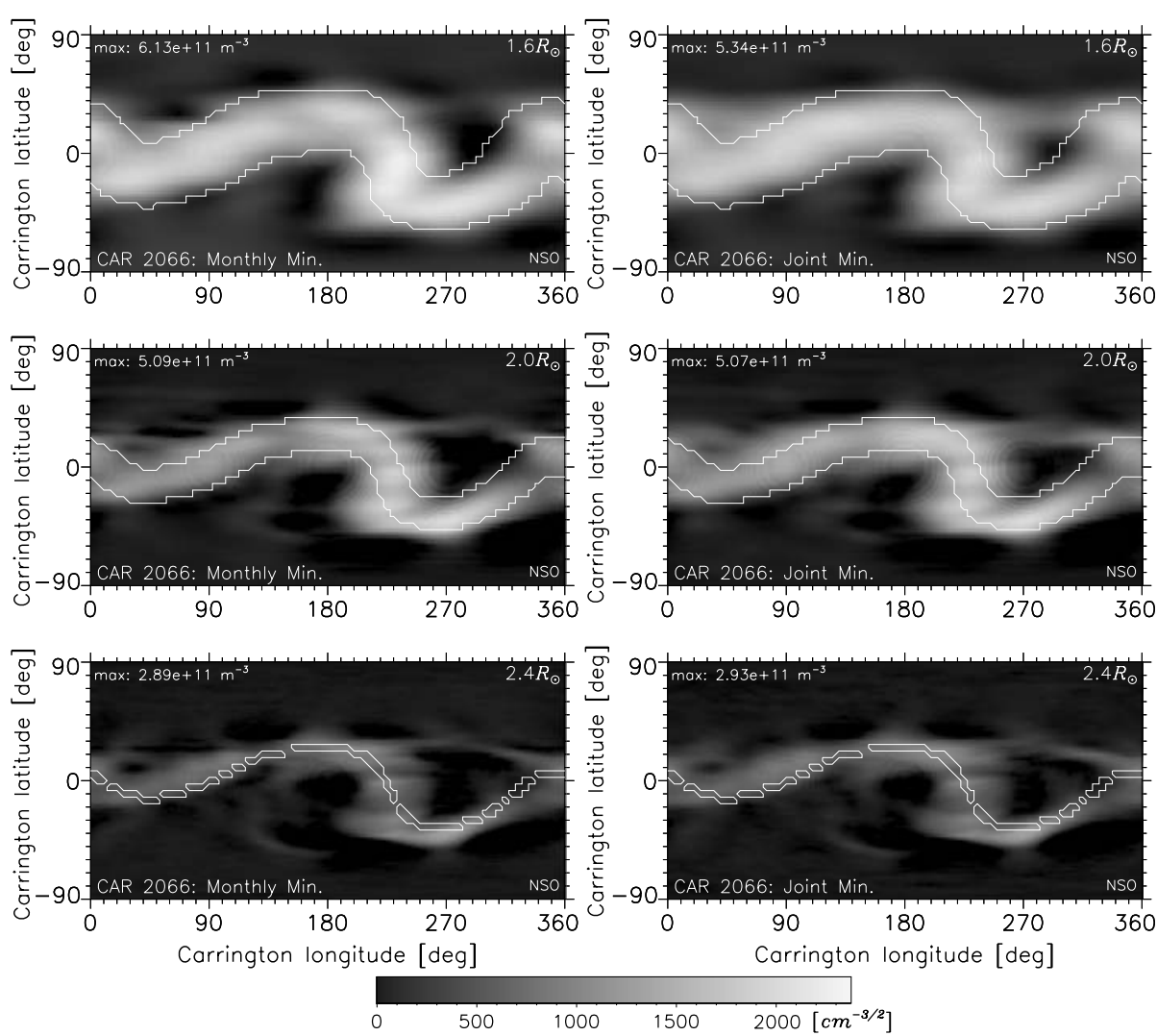

Figure 2 Spherical cross sections of the reconstructed electron density for CR 2066 at heliocentric distances $1.6 R_{\odot}, 2.0 R_{\odot}$, and $2.4 R_{\odot}$ (with distances shown in the right upper corners). The left panels correspond to a reconstruction using data processed with a MM background subtraction; the right panels correspond to the reconstruction based on the same data with JM background subtraction. The white contour lines are boundaries between closed and open magnetic field lines for the potential field approximation based on NSO/GONG data.

background made in one month for data from another month when maximum photometric accuracy is needed.

But the MM background is available for every month. Hence, a question arises: How does the choice of RM or MM background subtraction affect the electron density reconstruction?

To check this, we made two reconstructions for February 2008 (CR 2066), a month when a roll maneuver was conducted, one using the monthly and another using the JM background subtraction. Figure 2 shows spherical cross sections at different heights for these reconstructions. The two cross sections are very similar, with a difference in the local detail density values but not in the overall structure.

Figure 2 shows the boundaries between closed and open magnetic field lines in the potential field model with source surface (PFSS) at $R=2.5 R_{\odot}$ (Altschuler and Newkirk, 1969) and based on the National Solar Observatory (NSO) GONG data. ${ }^{1}$ The coefficients in the

\footnotetext{
${ }^{1}$ http://gong.nso.edu/data/magmap/QR/mqc/.
} 
Figure 3 Radial density profile through the streamer region with longitude $30^{\circ}$ and latitudes $-5^{\circ}$, $45^{\circ}$, and $75^{\circ}$. The reconstruction is for CR 2066 with JM background subtracted.

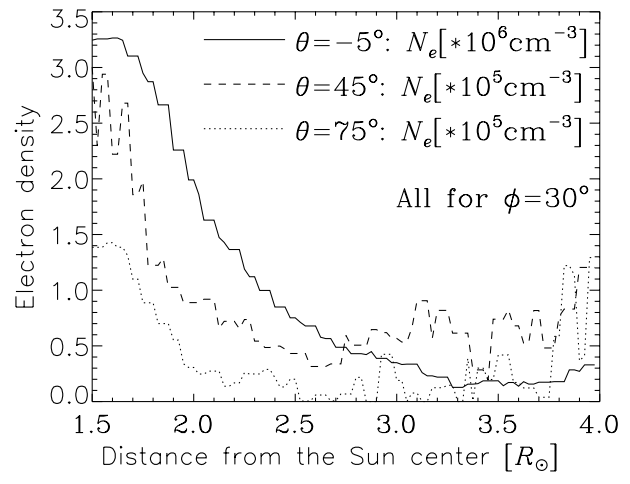

PFSS model are restricted to ninth order. It is clear that magnetic field structure follows the global density structure for this period (i.e., CR 2066).

This example shows that using the MM background subtraction for the electron density reconstruction to analyze the coronal density structure and behavior could be acceptable for obtaining the global density structure of the corona.

Figure 3 shows the density profile through the streamer region with longitude $30^{\circ}$ and latitude $-5^{\circ}$ for the reconstruction with JM background subtraction. At distances from $1.5 R_{\odot}$ to $\approx 1.7 R_{\odot}$ the density profile becomes constant. This could be caused by two factors: (1) the error in the vignetting correction, that is, when a portion of the beam passing near the occulter is blocked by the occulter, and (2) the principal limitation of tomography when precision of the tomographic reconstruction for the region near the occulter is lower (Natterer, 1986).

So, the difference between the real intensity value and the measured one is larger for rays near the occulter. This forces the reconstructed density value to decrease near the occulter during the inversion. Also at distances $\gtrsim 3.7 R_{\odot}$ the density increases. This is because the outer boundary of the reconstruction domain is a sphere with radius $4 R_{\odot}$ and the LOS integration path for rays near the outer boundary is relatively short, but a significant amount of the light contributed to the corresponding pixels comes from electrons outside the reconstruction domain. This effect also can be seen in Figure 6. The obtained values of the electron density are lower than those reported in Vasquez et al. (2008) using a similar method for LASCO/C2 data obtained during 21 April to 18 May 2005. There are two possible reasons for this: (1) reduced solar activity during the COR1 observations resulting in lower actual electron density or (2) uncertainty in the scattered light background subtracted from the COR1 images.

\section{Reconstruction for July 2007}

We also reconstruct the coronal electron density for the period of 3-16 July 2007, which corresponds to CR 2058. This period is characterized by a dark region near the equator (see Figure 4).

Monthly background images were subtracted to remove scattered light. As discussed in the previous section, the MM background subtraction also subtracts some of the K corona. Therefore our reconstruction does not give real values of the electron density, and we stress our analysis of density variations in the corona. Because this reconstruction was done before a new instrument calibration has been applied (see Table 3 in Thompson and Reginald, 2008), the actual density values could be higher by about 1.2 times in the reconstruction in addition to the other uncertainties just described. 
Figure 4 EUVI $195 \AA ̊$ image for 17 July 2007. Dotted lines are Carrington meridians and parallels with $30^{\circ}$ separation. The zero-value meridian and parallel are in the middle.
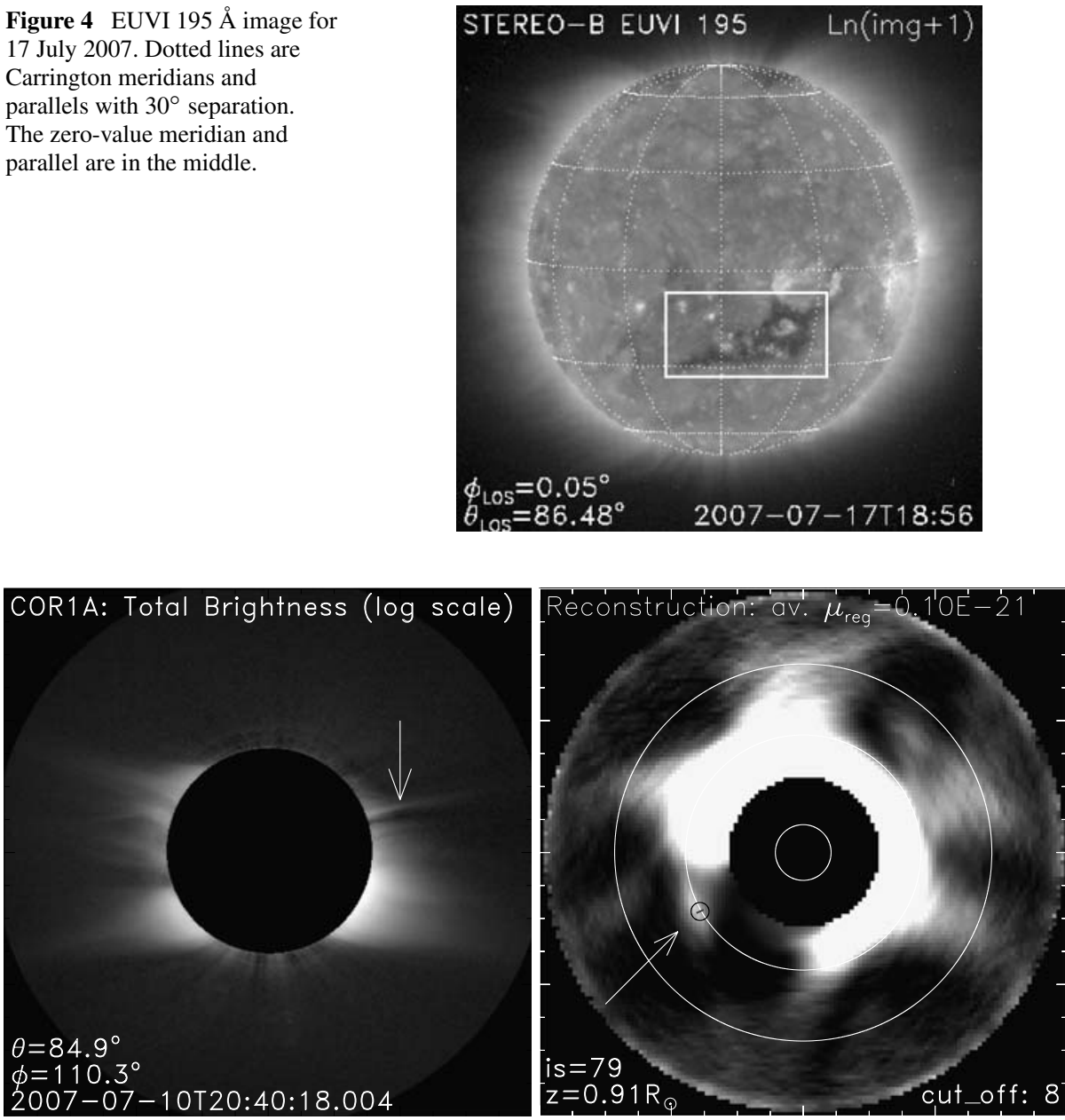

Figure 5 (Left) COR1 A total brightness image for 10 July 2007 at 20:40 UT on a logarithmic intensity scale. The streamer used for triangulation is marked by an arrow. (Right) The "horizontal" cross section of the reconstructed density at the level $z=0.91 R_{\odot}$. The small black circle with an arrow pointing to it is the position of the streamer obtained by triangulation.

\subsection{Reconstruction Result}

Because STEREO consists of two independent spacecraft observing the Sun from two different positions, triangulation can be used to find 3D coordinates of compact coronal structures (linelike or pointlike structures), and then the positions obtained from triangulation can be compared with the tomography reconstruction to verify the latter. To apply the triangulation method we identified an almost linelike streamer on both images from COR1 A and B. We show here only the COR1 A image with a narrow streamer marked by an arrow on the left panel of Figure 5. We use the Solar Soft IDL routine scc_measure for triangulation. The arrow in the right panel in Figure 5 marks the point where the coordinates were obtained by triangulation. The position of the streamer in the reconstruction is in agreement with the position found by triangulation. It shows that the method converges well. 

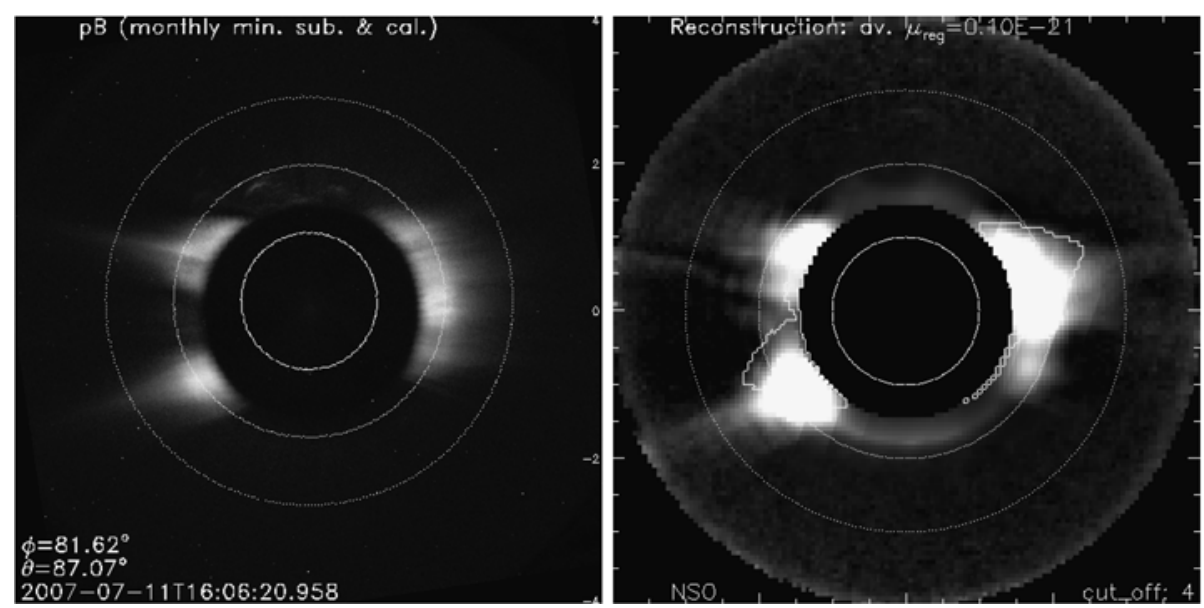

Figure 6 (Left) $\mathrm{pB}$ image corresponding to the viewing direction with Carrington longitude $81.6^{\circ}$ and colatitude $87.1^{\circ}$. (Right) Cross section of the reconstructed density by the plane perpendicular to the viewing direction of the image in the left panel. The contour line is the boundary between closed and opened field lines for the potential field approximation (CR 2058) based on NSO/GONG data.
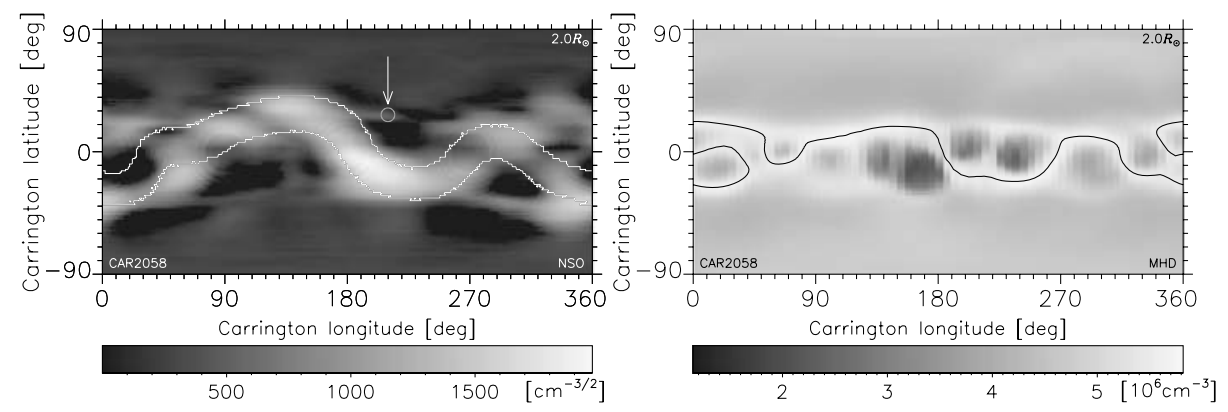

Figure 7 Spherical cross section at the distance $2 R_{\odot}$ for CR 2058. (Left) The reconstructed electron density. The white lines are boundaries between closed and open magnetic field lines for the potential field approximation based on NSO data. (Right) The electron density from the MHD solution. The black line is the neutral magnetic field line.

Figure 6 shows a comparison between an observed $\mathrm{pB}$ image and a corresponding cross section of the reconstructed electron density. The cross-section plane is perpendicular to the LOS direction for the observation. The brightness on the west side is widely spread in latitude, but the reconstruction shows that the density for the corresponding POS is more localized. This shows that not all the radiation comes from the POS.

Figure 7 (left) shows a comparison between the reconstructed density and potential magnetic field models with the source surface at $2.5 R_{\odot}$ based on the NSO/GONG data. The coefficients in the potential field model are restricted to ninth order. The figure shows that the magnetic field model correlates relatively well with the reconstructed density structure except for the region near zero longitude and $\sim 15^{\circ}$ latitude. The reconstructed density for this region is zero. Frazin and Kamalabadi (2005) argued that such zero-density values might be caused by temporal variation of the density there. However, a value of zero for the density in this particular region could also be because of the very low true density of this region, 
which is surrounded by much higher density structures, as is clearly seen on the STEREO-B EUVI image for the 19.5-nm bandpass corresponding to the Fe XII emission line (within the white rectangular box in Figure 4) at the same longitude and latitude as in the reconstruction. This huge difference in density in neighboring regions could cause a zero-density artifact in the reconstruction at the lower density region. To test this, a numerical experiment was conducted. We took this reconstructed density and set the density value in this zero-density region to $10^{3} \mathrm{~cm}^{-3}$. Then artificial data were produced with the same angular resolution as for the reconstruction with the real data, and the inversion was done in the same manner as with the real data. The reconstruction based on these simulated data shows the same zerodensity values in this region as the reconstruction with real data. This suggests that the large density contrast between low- and the high-density regions is likely responsible for the zero values in the reconstruction.

The arrow and circle in Figure 7 (left) marks the point corresponding to the coordinates obtained by triangulation of the streamer shown in Figure 5. This point coincides with the reconstructed density enhancement shown in Figure 7 (left). The feature chosen for triangulation has an extension in the meridional direction that influences the precision of the triangulation. However, because this extension is relatively small, the triangulation gives the point roughly in the middle of this feature.

Figure 7 (right) shows the result of the polytropic MHD model ${ }^{2}$ driven by the observed LOS photospheric magnetic field (Riley, Linker, and Mikic, 2001). The MHD model also shows some correlation with the reconstruction. However, additional comparisons are needed (see also Morgan, Habbal, and Lugaz, 2009, and Vasquez et al., 2008, for comparison of MHD simulations with other reconstruction methods) to quantify the differences.

\section{Conclusion}

In this paper we applied the regularized tomography method to STEREO-COR1 data and presented the first results obtained using this method. The regularized tomography reconstruction technique works well with COR1 data. The monthly minimum subtraction is satisfactory for analyzing the global structure of the corona. The reconstructed streamer belt position is mainly in agreement on the large scale with PFSS and MHD models. Moreover, positions of nearly linelike structures and several bright tips of helmet streamers are in agreement with their independent localization by triangulation. The zero-value density region in the reconstruction surrounded by streamers is clearly identified with the dark region in the EUVI image. This fact and also additional numerical simulations (see Section 4.1) give evidence that zero-density regions in the reconstruction are likely caused by regions of very low coronal density surrounded by more dense surrounding structures.

To increase the quality of the reconstruction, additional work is needed. First, higher resolution input images should be used; however, these would require increased computer resources. Also, using data from both STEREO spacecraft and Earth-based observations would allow us to reduce the stationarity assumption. In order to use multiple instruments in one reconstruction the instruments must be intercalibrated. ${ }^{3}$

We observe that the reconstruction error near the outer boundary of the reconstruction domain is higher. The LOS integration path for these distant rays is relatively short, and a

\footnotetext{
${ }^{2}$ http://www.predsci.com/stereo.

3 http://secchi-ical.wikidot.com/.
} 
significant amount of the light that contributes to the density derived for these pixels comes from electrons outside the reconstruction region. To reconstruct this region more precisely, it would be useful to use data from COR1 and COR2 instruments simultaneously.

A three-dimensional reconstruction of the type discussed in this paper could be produced for almost every Carrington rotation during the STEREO operational period, and then the reconstructions could be used as a test for coronal models.

Acknowledgements The authors are thankful to William Thompson and James McAteer for their help in data processing. MK thanks Gordon Petrie for useful comments about potential field reconstruction methods and Janet Luhmann whose potential field reconstruction code was used. Also, thanks are owed to Pete Riley and Jon Linker for the MHD simulation results and to Richard Frazin and Huw Morgan for comments that helped to improve the manuscript.

Open Access This article is distributed under the terms of the Creative Commons Attribution Noncommercial License which permits any noncommercial use, distribution, and reproduction in any medium, provided the original author(s) and source are credited.

\section{Appendix: Weighting Factor}

To demonstrate the influence of the weighting factor (Equation (5)) on the reconstruction results, a numerical experiment for a $2 \mathrm{D}$ scalar field tomography problem was performed.

Figure 8(a) shows the square root of the model 2D scalar field. The reconstruction domain is a square with side size equal to $8 R_{\odot}$. The background brightness of the model field decreases in a way similar to the electron density in the corona (Newkirk, Dupree, and Schmahl, 1970):

$$
N_{\mathrm{e}}\left(r_{s}\right) \propto\left(755 r_{s}^{-5.353}-168 r_{s}^{-14.738}+103800 r_{s}^{-20.446}\right),
$$

with $r_{s}=r+R_{0}$, where $r$ is the distance from the center of the image and $R_{0}=2$. The brightness of the round features is determined in the same way, but they are set to be brighter than the local background. The artificial data $y_{i}$ for the $i$ th ray were produced from this model field according to Equation (1) but with $K=$ const.

Then the inversion was made in the same way as described in Section 2 but without the weighting factor. The number of observing directions is set to 30 . The reconstruction domain is a $512 \times 512$ rectangular grid. The results for two values of $\mu$ are presented on Figures 8(b) and (c). Radial artifacts are clearly seen owing to the bright central region and small number of observing directions.

Next an inversion with a weighting factor was made. The weighting factor was produced as in Equation (5). The artificial data for the same set of rays were produced by using the model field without round features (i.e., by taking into account only background). Thus, the weighting factor for the $i$ th ray is

$$
w_{i}=\frac{1}{y_{i}^{(\mathrm{bkg})}-\min \left(y_{i}^{(\mathrm{bkg})}\right) / 10} .
$$

Division by 10 is introduced here to prevent possible division by zero. So, the minimizing function now is

$$
F=\sum_{i}\left[\left(\sum_{j} w_{i} a_{i j} x_{j}\right)-w_{i} y_{i}\right]^{2}+\mu F_{\mathrm{reg}}(\mathbf{X}) .
$$



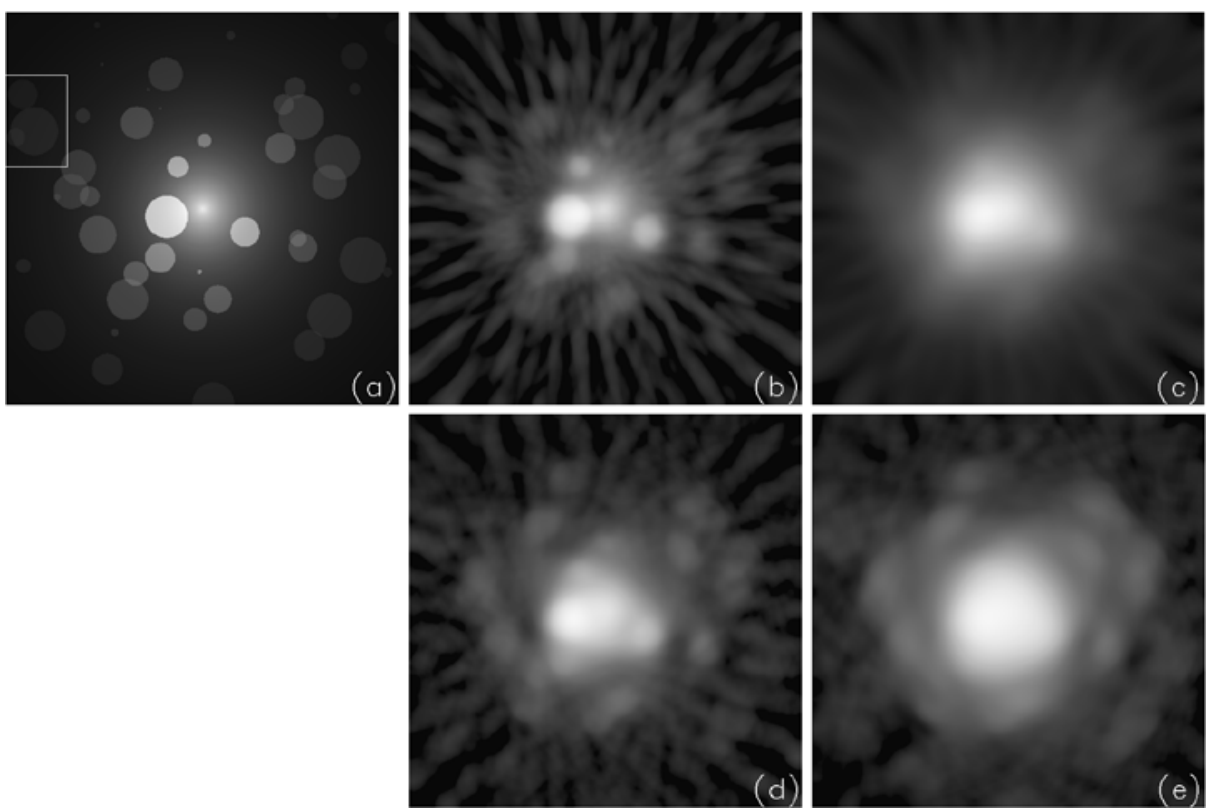

Figure 8 Reconstruction of 2D scalar field: (a) original model; (b), (c) reconstruction without weighting factor for two different values of the regularization parameter; (d), (e) reconstruction with weighting factor for two different values of the regularization parameter.

The results of the reconstruction for two values of $\mu$ with the weighting factor are shown on Figures 8(d) and (e). The reconstruction with the weighting factor does show reduced radial artifacts compared to the reconstruction without the weighting factor. Moreover, the region inside the white rectangular box (marked in Figure 8(a)) is seen in the reconstruction with the weighting factor (especially clear in Figure 8(e)), but not on the reconstruction without the weighting factor. The reader can find more round features that are seen in the reconstruction with the weighting factor but not seen in the reconstructions without it (for example, in the lower left corner). However, the central background part is more smeared.

This example shows that the weighting factor in Equation (5) is useful for regularized tomography; however, more detailed study is needed.

The weighting factor works like the regularization parameter $\mu_{\text {tomo }}$ for tomography term $F_{\text {tomo }}$ in the minimization function (8) when $\mu_{\text {tomo }}$ depends on ray $i$.

A weighting factor that acts in a similar way can also be seen in Equation (4) of Wiegelmann and Inhester (2003). The role of the weighing factor is played there by the multiplier $B^{-2}$.

When $K$ very rapidly decreases with distance from the Sun, the weighting factor could be chosen according to the Jacobi preconditioning as in Equation (14) of Kramar and Inhester (2006).

\section{References}

Altschuler, M.D., Newkirk, G.: 1969, Solar Phys. 9, 131.

Billings, D.E.: 1966, A Guide to the Solar Corona, Academic Press, New York.

Blackwell, D.E., Petford, A.D.: 1966a, Mon. Not. Roy. Astron. Soc. 131, 383. 
Blackwell, D.E., Petford, A.D.: 1966b, Mon. Not. Roy. Astron. Soc. 131, 399.

Davila, J.M.: 1994, Astrophys. J. 423, 871.

Frazin, R.A., Janzen, P.: 2002, Astrophys. J. 570, 408.

Frazin, R.A., Kamalabadi, F.: 2005, Solar Phys. 228, 219.

Frazin, R.A., Vasquez, A.M., Kamalabadi, F., Park, H.: 2007, Astrophys. J. 671, L201.

Inhester, B.: 2006, Publ. Int. Space Sci. Inst. to appear. astro-ph/0612649.

Kramar, M., Inhester, B.: 2006, Astron. Astrophys. 456, 665.

Moran, T.G., Davila, J.M., Morrill, J.S., Wang, D., Howard, R.: 2006, Solar Phys. 237, 211.

Morgan, H., Habbal, S.R., Lugaz, N.: 2009, Astrophys. J. 690, 1119.

Natterer, F.: 1986, The Mathematics of Computerized Tomography, Wiley, Chichester.

Newkirk, G. Jr., Dupree, R.G., Schmahl, E.J.: 1970, Solar Phys. 15, 15.

Panasyuk, A.V., Strachan, L., Finesehi, S., Gardner, L.D., Raymond, J., Kohl, J.L. et al.: 1998, In: Balasubramaniam, K.S., Harvey, J., Rabin, D. (eds.) ASP Conf. Ser. 140, 407.

Quemerais, E., Lamy, P.: 2002, Astron. Astrophys. 393, 295.

Riley, P., Linker, J.A., Mikic, Z.: 2001, J. Geophys. Res. 106, 15889.

Tikhonov, A.N.: 1963, Sov. Math. Dokl. 4, 1035.

Thompson, W.T., Reginald, N.L.: 2008, Solar Phys. 250, 443.

Van de Hulst, H.C.: 1950, Bull. Astron. Inst. Neth. 11, 135.

Vasquez, A.M., Frazin, R.A., Hayashi, K., Sokolov, I.V., Cohen, O., Manchester, W.B. IV, Kamalabadi, F.: 2008, Astrophys. J., 682, 1328.

Wiegelmann, T., Inhester, B.: 2003, Solar Phys. 214, 287.

Zidowitz, S.: 1999, J. Geophys. Res. 104, 9727. 